\title{
PLASMA SURFACE MODIFICATION OF POLYSTYRENE AND POLYETHYLENE
}

\author{
S.Guruvenket, G.Mohan Rao*, Manoj Komath ${ }^{1}$ and Ashok.M.Raichur ${ }^{2}$ \\ Department of Instrumentation, Indian Institute of Science, Bangalore 560 012. India \\ ${ }^{1}$ Biomedical wing, Sree Chithra Tirunal Institute of Medical Science and Technology, \\ Thiruvandrum 695 012. India. \\ ${ }^{2}$ Department of Metallurgy, Indian Institute of Science, Bangalore 560 012. India
}

\begin{abstract}
Polystyrene (PS) and polyethylene (PE) samples were treated with argon and oxygen plasmas. Microwave Electron Cyclotron Resonance (ECR) was used to generate the argon and oxygen plasmas and these plasmas were used to modify the surface of the polymers. The samples were processed at different microwave powers and treatment time and the surface modification of the polymer was evaluated by measuring the water contact angle of the samples before and after the modification. Decrease in the contact angle was observed with the increase in the microwave power for both polystyrene and polyethylene. Plasma parameters were assessed using Langmuir probe measurements. Fourier transform infrared spectroscopy showed the evidence for the induction of oxygen based functional groups in both polyethylene and polystyrene when treated with the oxygen plasma. Argon treatment of the polymers showed improvement in the wettability which is attributed to the process called as CASING, on the other hand the oxygen plasma treatment of the polymers showed surface functionalization. Correlation between the plasma parameters and the surface modification of the polymer is also discussed
\end{abstract}

*Corresponding author: gmrao@isu.iisc.ernet.in 
Key words: Surface modification, polyethylene, polystyrene, ECR plasma

\section{INTRODUCTION}

Control of surface properties is very important for the high performance of adhesion, biomaterials and paints. Surface modification of hydrophobic polymer surface into a hydrophilic or vice versa can be achieved by wet (acid, alkali), dry (plasma) and radiation treatments (ultraviolet radiation and laser) with out affecting the bulk properties [1]. Glow discharge plasmas are generally used for the surface modification of polymers because the processes involved are solvent free and dry, the consumption of chemicals is extremely low and need for sterilization of the products is eliminated. Moreover these processes are precisely controllable. The surface can be treated homogeneously and the surface chemistry can be tailored for the required end use [2]. Glow discharge plasmas contain energetic neutrals, ions and electrons, which act on a surface under treatment to change its physico-chemical properties. The nature of the gas species used and the energy of the ions are important parameters, which can affect the properties of the polymer surface immersed in plasma. Glow discharge plasmas are created by the inducing an electric potential or electromagnetic radiation (radio frequency or microwaves) at lowpressures [2].

To understand the complex reaction between the plasma and the polymer surface, it is important to understand the plasma parameters and their effect on the surface modification. In most of the reports the plasma excitation source power (RF or Microwave) is compared with extent of surface modification, but in practice it is not only the excitation power but also the pressure, degree of ionization and other plasma parameters that affect the polymer surface. Hence it is important to consider the effect of all the other plasma properties (microscopic plasma parameters, like ion energy, ion flux, degree of ionization etc.) to analyze the surface modification of polymers.

In our current investigation we used ECR plasma source to modify the surface of polyethylene and polystyrene samples. The ECR plasma has significant advantage over other techniques like Radio Frequency Glow Discharge (RFGD) 
frequently used in polymer surface modifications (3). ECR sources can be operated at low pressures $\left(10^{-4}\right.$ to $10^{-3} \mathrm{mbar}$ ), which reduces the risk of gas phase contamination during processing. The ion densities are higher (of the order of $10^{11}$ to $10^{13} \mathrm{per} \mathrm{cm}^{3}$ ) and the energy of the plasma species is moderate $(<50 \mathrm{eV})$. This combination of higher ion density and lower ion energy ensures a better surface modification of the polymer compared with the conventional plasma surface modification techniques. Moreover the plasma properties can be controlled conveniently by manipulating the strength and configuration of the magnetic field in the source chamber and by adjusting the microwave power.

Polystyrene and polyethylene are commonly used polymers in the biomedical applications and are generally hydrophilic in nature. Certain applications like cell culture discs $[4,5]$ require more hydrophilic surface, with out altering the bulk properties of the polymers. Hence surface modification of these polymers becomes important. In this report we demonstrate the effect of ECR plasma on the surface property of polyethylene and polystyrene with argon (inert) and oxygen (reactive).

\section{Experimental}

Electron Cyclotron Resonance (ECR) technique whose description is available elsewhere [6] is used to modify the polymer surfaces. The schematic diagram of the ECR source is shown in the Figure1. It consists of a source chamber with two electromagnetic coils wound around and a processing chamber underneath, both connected to a vacuum system. Microwave power generated in a continuous wave magnetron source $(2.45 \mathrm{GHz})$ is fed from the top through a quartz window with appropriate wave-guides. The electromagnets are positioned to get a magnetic mirror inside the source chamber. The gas (either argon or oxygen) is admitted in to the source chamber using a flow controller and applying the microwave power generates glow discharge. The downstream plasma extended to the processing chamber is used for the surface modification.

The chambers are evacuated to a base pressure of $10^{-6} \mathrm{mbar}$ and the working pressure is maintained at $10^{-3}$ mbar by admitting the appropriate gas. The distance between the source and the sample is maintained at $10 \mathrm{~cm}$. The currents in the 
upper and the lower magnetic coils are maintained at 60A. The effect of surface treatment is studied systematically by changing the microwave power and the processing time with Argon $(99.999 \%)$ and oxygen $(99.99 \%)$ as the processing gases. Biomedical grade polystyrene and polyethylene samples have been cut into small pieces $(1 \mathrm{~cm} \times 1 \mathrm{~cm})$ and ultrasonically cleaned in a doubly dionized water prior to surface modification treatments.

The modification is carried out at different microwave powers and different processing times. The plasma parameters were diagnosed using Langmuir probe set up and the surface modification of the polymers was studied using water contact angle measurements and the FTIR-ATR analysis [7]. After processing, the treated polymer samples were transferred to a vacuum decicator to avoid the absorption of the moisture from the atmosphere. Surface modification can be easily characterized by measuring contact angles of the surface with liquids [8]. In the present study, sessile drop technique is used under ambient atmospheric conditions. Further measurements were carried out on these samples. The contact angle measurements were carried out in ambient atmosphere. FTIR ATR experiments were carried out using Impact 410 spectrometer with a resolution of $1 \mathrm{~cm}^{-1}$, treated and untreated polymer surface are kept pressed against ATR crystal (Germanium) and the spectra were recorded. The maximum penetration depth of incident radiation at the incidence angle of $60^{\circ}$ for polystyrene and polyethylene with Germanium crystal is $\sim 610 \mathrm{~nm}$. The contact angle measurement and FTIR-ATR experiments were carried out ex situ under ambient conditions.

\section{RESULTS AND DISCUSSION}

\section{Langmuir probe analysis}

Plasma parameters like ion density, electron temperature etc., play a vital role in the surface modification of the polymers. Hence it is necessary to determine the plasma parameters to have a better understanding on the basic reaction occurring between the polymer and plasma. In the present investigation Langmuir probe is used to measure the plasma parameters. Langmuir probe basically consists of a cylindrical probe 
(tungsten wire of $0.25 \mathrm{~mm}$ diameter and $5 \mathrm{~mm}$ length) placed perpendicular to the plasma stream. Langmuir probe is placed at the same position where the polymer samples are placed for the surface treatment. A potential is imposed on the metal probe relative to the ground that is in contact with plasma. In the present study the bias was varied from $-60 \mathrm{~V}$ to $60 \mathrm{~V}$ volt and the probe current was measured. Ion density and plasma potential were determined from the curve of the I-V plot using standard methods [9], which is based on the assumptions that ions and electrons in the ECR plasma follow Maxwellian distribution. Figure 2 shows the variation in the plasma potential $\left(\mathrm{V}_{\mathrm{p}}\right)$ and ion density $\left(\mathrm{N}_{\mathrm{i}}\right)$ as the function of microwave power. It is seen from the diagram that ion density increases with the microwave power, where as, the plasma potential decreases with the same. Beyond 300 watts of microwave power the change in plasma potential becomes rapid. This indicates the increase in the ion energy and the ion density beyond 300Watts. Ion energy is an important parameter, which is responsible for the induction of the chemical reaction and the surface heating effect, which could lead to deterioration of the polymer surface. It was also found that the higher ion energy increases the roughness of the polymer surface, which affects the contact angle measurements [10,11]. It is found that there is no appreciable change in the ion density and in the plasma potential when the plasma gas is changed from Argon to oxygen An optimum combination of ion energy and ion density will favor the chemical activation on the surface of the polymer rather increasing the surface roughness or deteriorating the surface/ bulk properties of the polymer. During our earlier studies it was observed that at microwave powers of more than 250 watts and higher treatment times (more than 180 seconds) deteriorate the polymer surface and hence in our present investigations, experiments were carried out at lower microwave power to ensure surface modification without affecting the bulk properties and the surface morphology.

\section{CONTACT ANGLE MEASUREMENTS}

Contact angle of the polymer surface was determined before and after the treatment using DDI (doubly dionized) water. Teflon (PTFE) and glass (standard hydrophobic and hydrophilic materials) were used to validate the measurements. The measured contact angles of these samples were $110^{\circ}$ and $14^{\circ}$ respectively which agrees 
with the reported values $[8,12]$. The water contact angles of the polystyrene samples treated with argon and oxygen plasmas at different microwave power and treatment time are shown in the table (1). Similarly water contact angles of the polyethylene samples treated with the argon and oxygen at different plasma power and treatment time were determined and are shown in the table (2). These changes in contact angle due to plasma treatment have been discussed later.

\section{FTIR-ATR analysis}

Plasma surface modifications are confined only to a few nanometers below the surface. FTIR spectroscopy in Attenuated Total Reflection (ATR) mode is one of the methods used to bring out the finer surface information [7]. We used this technique to characterize the polymer surface. The spectra of the treated and the untreated samples are compared to observe the changes.

Figure 3 shows the FTIR ATR spectra of untreated polystyrene and that treated with argon and the oxygen plasma. When the untreated polystyrene spectrum is compared with the argon treated polystyrene spectrum it is seen that a broad band occurs around $3300 \mathrm{~cm}^{-1}$ and at $1600 \mathrm{~cm}^{-1}$, which correspond to the hydroxyl group (-OH). Similarly the polyethylene samples treated with the argon plasma (figure 4) when compared with the untreated polyethylene shows peaks at $3300 \mathrm{~cm}^{-1}, 1737 \mathrm{~cm}^{-1}$, $1646 \mathrm{~cm}^{-1}$ and $1535 \mathrm{~cm}^{-1}$. Contact angle measurements on these treated surfaces of polystyrene and polyethylene samples (shown in the table 1 and table 2) indicate the hydrophilic transformation of the polymer surfaces.

When the surface of polymer is treated with the inert gas plasmas, it is observed that the low molecular weight chains on the surfaces are detached and in turn help in improving the cross linkage density and hence the surface is activated. When inert gas plasma treated polymer samples are exposed to the atmosphere after the treatment the plasma activated surface readily adsorbs the moisture that is present in the environment which is indicated by the broad band at $3300 \mathrm{~cm}^{-1}$ and $1600 \mathrm{~cm}^{-1}$ in case argon plasma treated polystyrene and at $3300 \mathrm{~cm}^{-1}$, in the case of polyethylene. Additional peaks at 
$1737 \mathrm{~cm}^{-1}$ correspond to $(\mathrm{C}=\mathrm{O})$ stretching vibration and the band at $1646 \mathrm{~cm}^{-1}$ corresponds to $\mathrm{COO}^{-}$asymmetrical stretching and the one at $1368 \mathrm{~cm}^{-1}$ (broadening of the peak) may be due to the $\mathrm{COO}^{-}$symmetrical stretching. These additional peaks show the post treatment functionalization of the polyethylene surface. The exposure of the polymer to the inert gas plasma ( in the present case argon) is sufficient to abstract hydrogen and to form free radicals at or near the surface which then interact to form the cross-links and unsaturated groups with the chain scission . The plasma also removes the low molecularweight materials or converts them to a high-molecular weight by cross-linking reactions. As a result, the weakly bound layers formed by the low molecular weight materials are removed. This treatment has been known as CASING (cross-linkage by activated species of inert gases) $[13,14]$. The plasma analysis (Figure 2) indicates that with increase in the microwave power the ion density also increases whereas the plasma potential decreases with the increase in the microwave power. Plasma treatment of a polymer surface requires high-density low energy ions in the plasma. ECR plasma contains the low energy and high-density ions, which is most preferred for a polymer surface treatment. And also it should be noted that with the increase in the microwave power the contact angle value on the treated surface of polystyrene and polyethylene decreases, while the ion density increases with the increase in microwave power. It is also observed that the prolonged treatment for a longer periods or at higher microwave power, detoriates the surface of the polymer and hence the treatment of the polymer is confined with in a certain microwave power and the treatment time [15].

The treatment of polystyrene and polyethylene with the oxygen plasma has a different effect when compared to that of the Argon treated samples. In addition to the surface activation of polymer surface, functionalization of polymer occurs due to the active nature of the plasma gas used (in present case it is Oxygen). In case of polystyrene treated with oxygen plasma (figure 3 ) the peak at $1746 \mathrm{~cm}^{-1}$ corresponds to $\mathrm{C}=\mathrm{O}$ stretching, which indicates that the surface is not only getting activated but also the plasma reaction of oxygen with the surface to form a $\mathrm{C}=\mathrm{O}$ group on the surface. This effect is responsible for the decrease in the contact angle that occurs at much lower microwave power/ treatment time than the argon treated polystyrene surface. Meichsner 
et al observed bands at $\left(3500 \mathrm{~cm}^{-1}\right.$ to $\left.3000 \mathrm{~cm}^{-1}\right)$ and various C-O bands $\left(1300 \mathrm{~cm}^{-1}\right.$ $1000 \mathrm{~cm}^{-1}$ ), which are similar to our observations [16]. In figure 4 (polyethylene treated with oxygen), the band around $3500 \mathrm{~cm}^{-1}$ is due to the (-OH) group and the broadening could be due to its intermolecular or intramolecular reactions. The band at $1741 \mathrm{~cm}^{-1}$ is due to $\mathrm{C}=\mathrm{O}$ group. The band around $1693 \mathrm{~cm}^{-1}$ is due to $\mathrm{COO}^{-}$asymmetrical stretching and at $1372 \mathrm{~cm}^{-1}$ could be due to symmetrical stretching of $\mathrm{COO}^{-}$and the band around $1100 \mathrm{~cm}^{-1}$ could be due to $\mathrm{C}-\mathrm{O}$ stretching. The C-O stretching from C-C-O appears at $1030-1110 \mathrm{~cm}^{-1}$ depending on the substituents. In this case it appears at $1100 \mathrm{~cm}^{-1}$. Marian Lehocky et al has also observed similar kind of group attachment on the surface on polyethylene samples treated with oxygen plasmas [17]. It is well known that an oxygen plasma can react with the polymer surface to produce a variety of atomic oxygen functional groups, including $\mathrm{C}-\mathrm{O}, \mathrm{C}=\mathrm{O}, \mathrm{O}-\mathrm{C}=\mathrm{O}$ and $\mathrm{CO}_{3}$ at the surface. In an oxygen plasma, two processes occur simultaneously: viz., etching of the polymer surface through the reaction of atomic oxygen with the surface carbon atom, giving volatile reaction products and the other one is the formation of oxygen functional groups at the polymer surface through the interaction between the active species from the plasma and the surface atom [18]. The balance of these two processes depends on the operation of a given process. The contact angle measurements done on the oxygen plasma treated polystyrene and polyethylene samples indicate the fast transformation to the hydrophilic nature compared to the polyethylene and polystyrene samples treated with the argon plasmas. It can also be observed that the treatment time /power required to bring down the contact angle using oxygen plasma is much less than the treatment time/power required to bring down the contact angle using argon plasma.

\section{CONCLUSIONS}

In this investigation it was found that the ECR plasma treatment of the Polystyrene and Polyethylene with oxygen and argon is an effective tool for improving the wettability. The ECR plasma analysis clearly indicates the increase in the ion density and decrease in the ion energy with increase in the microwave power. Surface 
modification of the polymers was carried out at different microwave powers and treatment times. It was found that the contact angle with water decreases with both microwave power and treatment time in argon as well as oxygen plasma. Argon treated polymers showed improvement in the wettability which is attributed to the process called as CASING, FTIR ATR analysis of the polymer samples treated in argon plasma showed absorption of moisture. FTIR ATR analysis of oxygen treated polymer surface shows several oxygen based functionalities at the surface (carbonyl, carboxyl, ether, peroxide etc). Thus this work demonstrates the use of ECR plasma source with oxygen and Argon plasmas to modify the surface of the polyethylene and polystyrene.

\section{References}

[1] S.L. Kapalan and P.W. Rose, Intl. Journal of Adhesion and Adhesives, 11 (1991) 109

[2] C.M.Chan, T.M. Ko and H. Hiraoka, Surface Science and reports, 24 (1996) 1.

[3] S, Nowak, P.Groning, O.M. Kuttel, M.Colland and G.Diltler, J.Vac. Sci. Technol., A $10(1992) 6$

[4] R.I Freshney, in Culture of Animal Cells ( ${ }^{\text {rd }}$ edition), Wiley, New York (1994) P- 71 .

[5] P.Larocca, Cell Line, 6 (1996) 2.

[6] K.Deenama Varghese and G.Mohan Rao, Rev. Sci Instru., 71, (2000) 407

[7] R. Rochotzki, M. Nitschke, M.Artz and J.Meischsner, Phys. Stat. Sol (a) 145, (1994) 289

[8] A.W, Adamson in Physical chemistry of surfaces ( $3^{\text {rd }}$ edition) wiley, New York (1976)

[9] B.N.Chapman, in Glow Discharge process, Wiley, New York. (1980) 
[10] G.S.Tzeng, H.S. Chen, Y.Y. Wang and C.C. Wab, Surf. and Coat. Technol., 89 (1997) 108.

[11] D.W.Dwight and W.N. Riggs, J. Coll and Interf. Sci., 47 (1974) 650

[12] R.Dann, J Coll and Interf. Sci., 32 (1970) 302

[13] R H Hansen and H. Schonhorn , J. Poly. Sci., B 4 (1966) 203.

[14] H. Schonhorn and R.H.Hansen, J.Appl. Poly. Sci.,11 (1967) 1461.

[15] S. Guruvenket, Manoj Komath, S.P. Vijayalakshmi, A.M. Raichur and G.Mohan Rao, J. Appl. Poly. Sci. 90, (2003) 1618-1623.

[16] J.Meichsner, M.Zeuner, B.Kramers, M.Nitschke, R.Rochotzki, K. Barucki Surf and Coat. Technol. 98 (1998) 1565.

[17] Marian Lehocky, Hana Drnovski, Barbora Lapcikova, A.M. Barros Timmons Tito Trindade. Maria Zembala, Lubomir Lapcik, Jr, Colloids and Surfaces -A $22(2003) 125$

[18] M. Morra E.Occhiello and F.Garbassi, Surf. Interf Anal., 16 (1990) 412. 


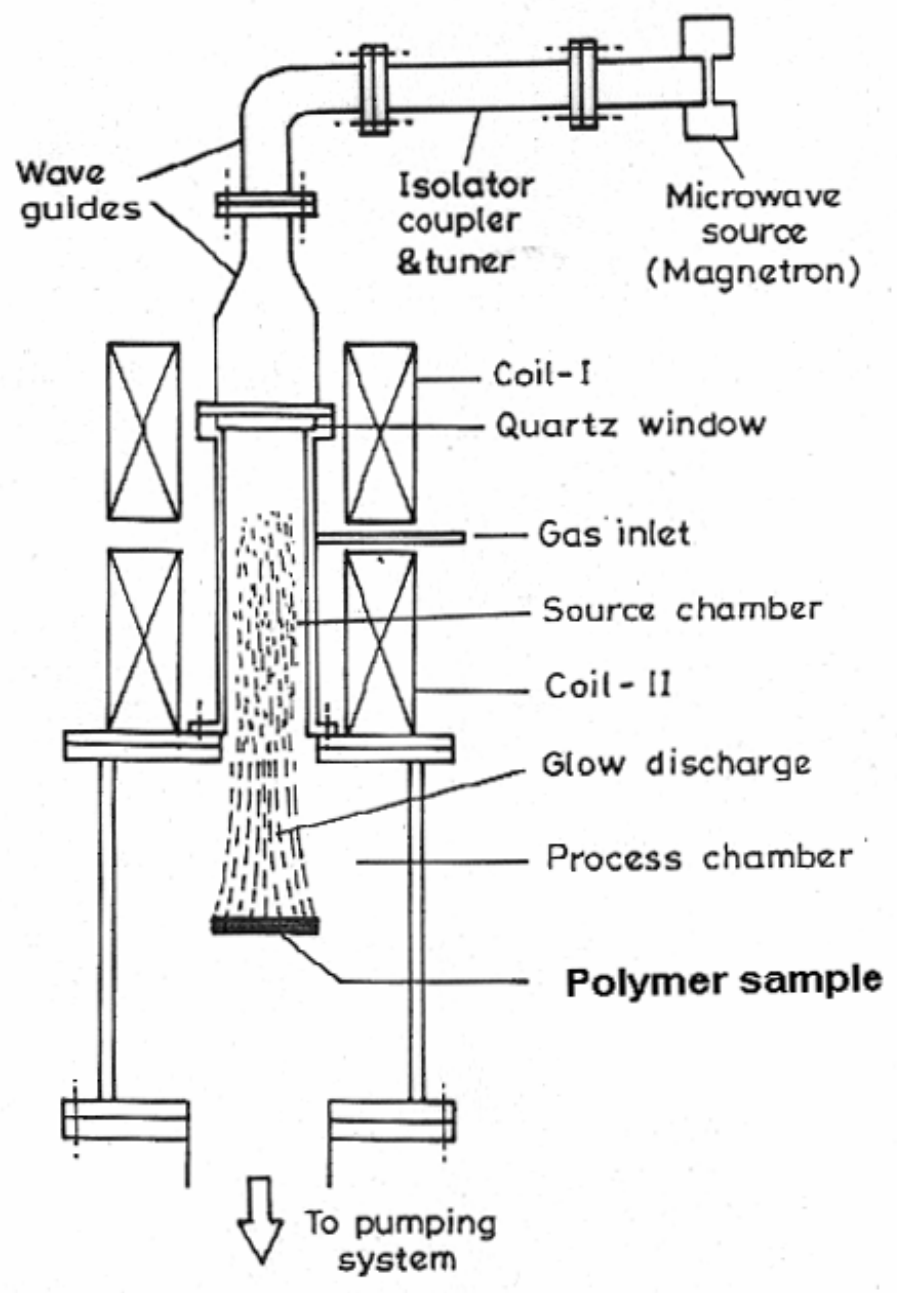

Figure 1 : Schematic of Electron Cyclotron Resonance (ECR) Plasma Chamber 


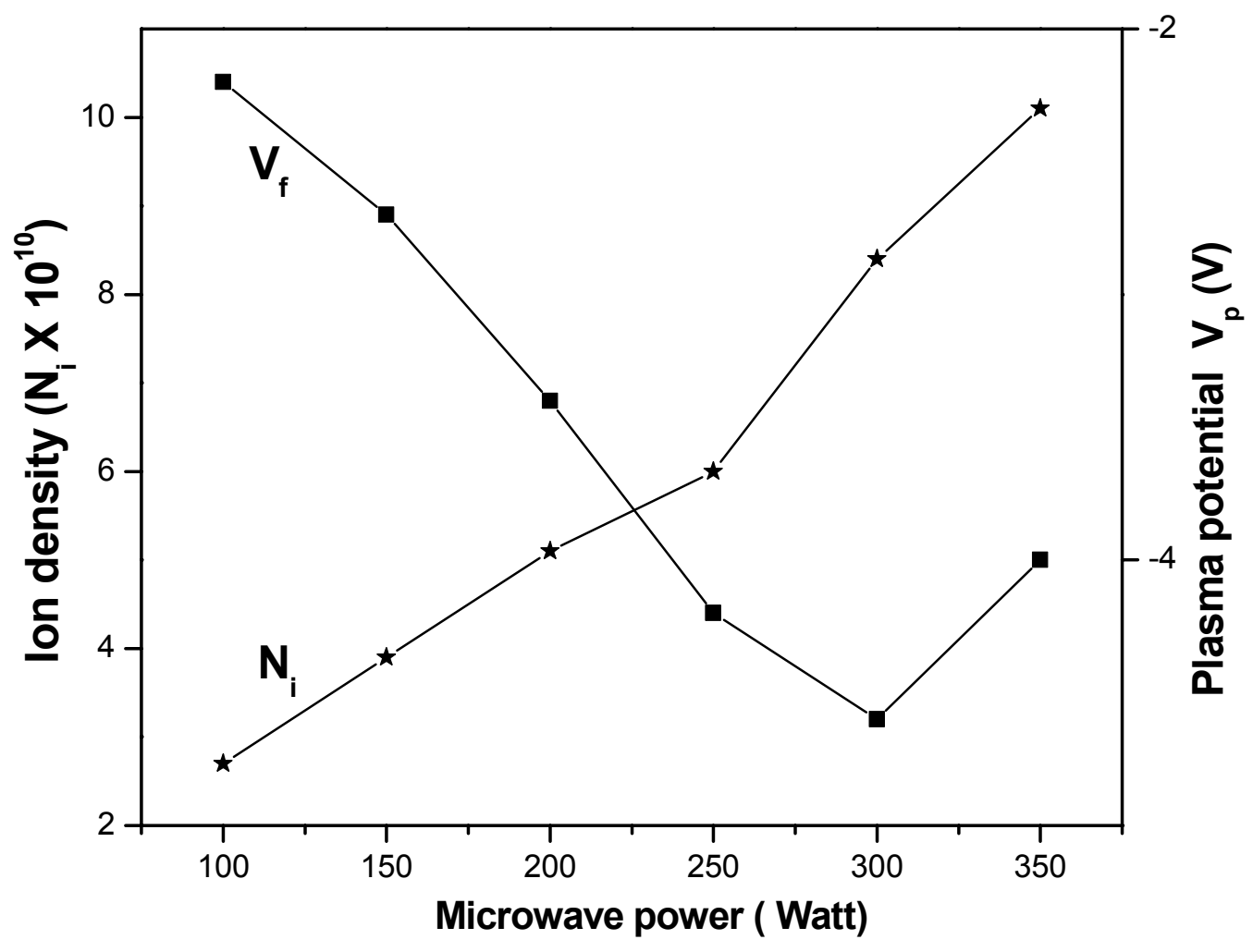

Figure 2: Variation of the plasma potential $\left(\mathrm{V}_{\mathrm{p}}\right)$ and ion density $\left(\mathrm{N}_{\mathrm{i}}\right)$ as the function of the microwave power for argon plasma 


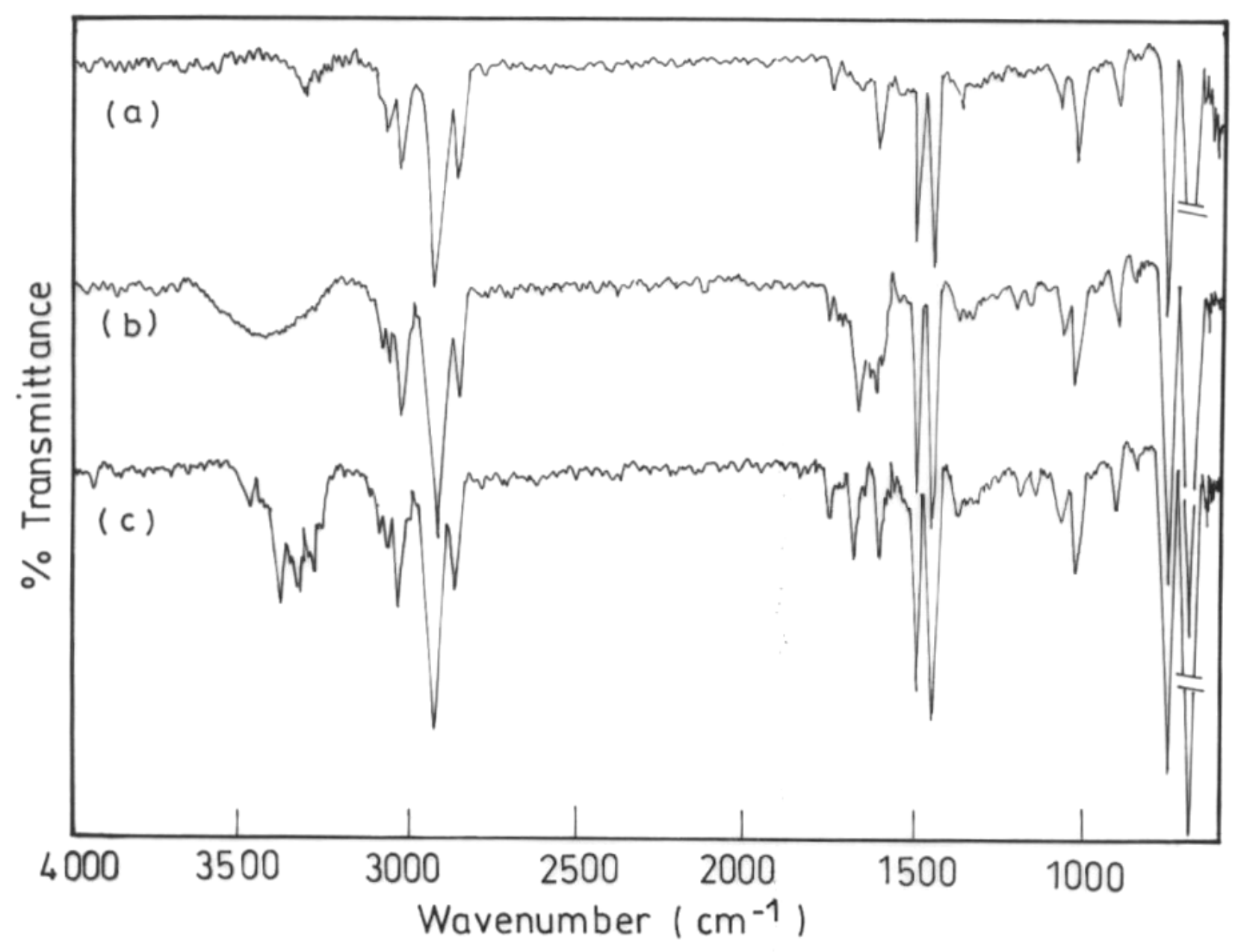

Figure(3) : FTIR Spectra of Polystyrene treated with Argon and Oxygen plasma.

(a) Untreated

(b) 200 Watt, 120 Seconds ( argon plasma)

(c) 200 Watt, 120 Seconds ( oxygen plasma) 


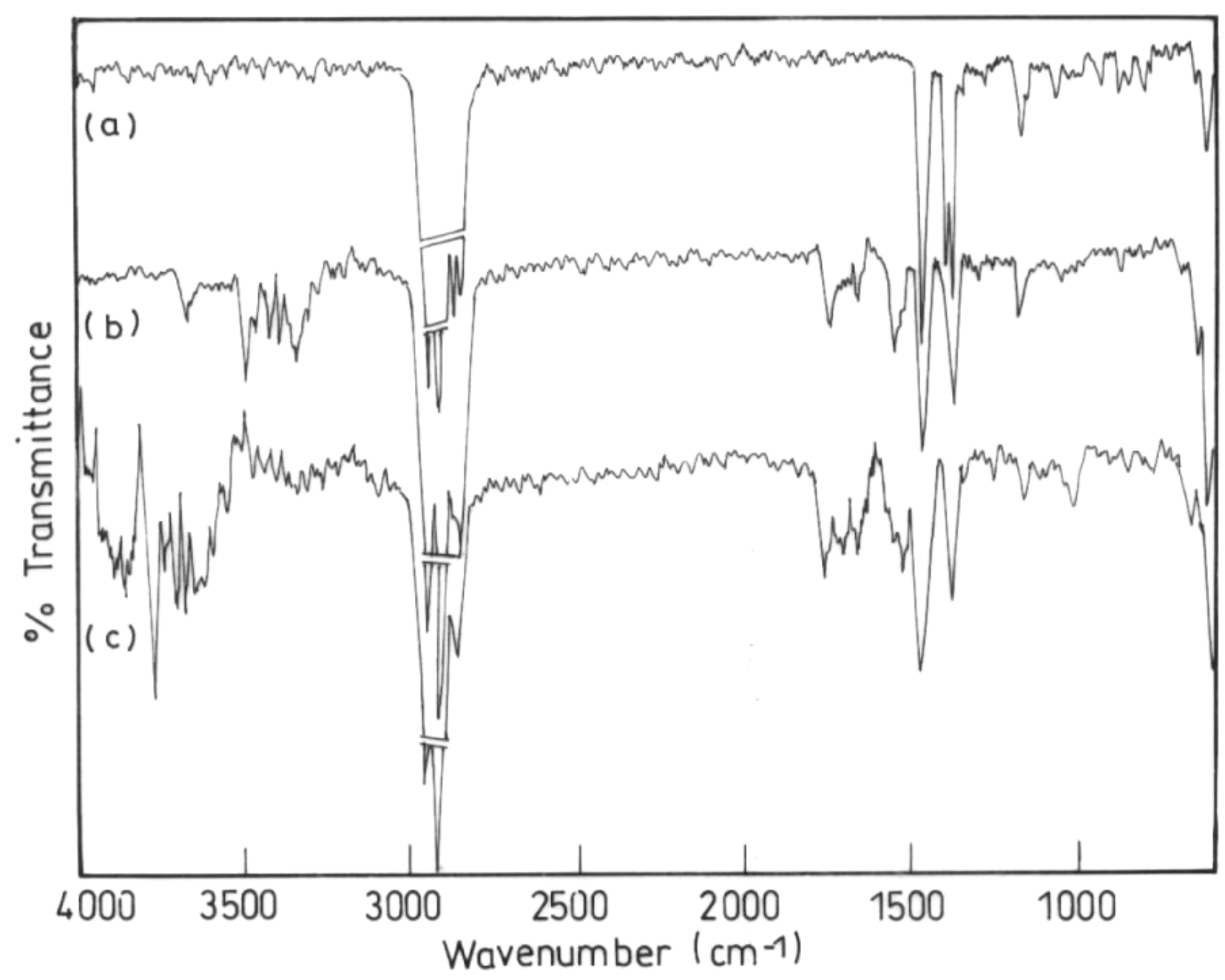

Figure(4) : FTIR Spectra of Polyethylene treated with Argon and Oxygen plasma.

(d) Untreated

(e) 200 Watt, 120 Seconds ( argon plasma)

(f) 200 Watt, 120 Seconds ( oxygen plasma) 


\begin{tabular}{|c|c|c|c|c|c|c|}
\hline \multirow{2}{*}{\begin{tabular}{c} 
Treatment \\
\multirow{2}{*}{$\begin{array}{c}\text { time } \\
\text { seconds) }\end{array}$}
\end{tabular}} & \multicolumn{3}{|c|}{$\begin{array}{c}\text { Argon plasma treated } \\
\text { Microwave power (watt) }\end{array}$} & \multicolumn{3}{c|}{$\begin{array}{c}\text { Oxygen plasma treated } \\
\text { Microwave power (watt) }\end{array}$} \\
\cline { 2 - 7 } & $\mathbf{1 0 0}$ & $\mathbf{1 5 0}$ & $\mathbf{2 0 0}$ & $\mathbf{1 0 0}$ & $\mathbf{1 5 0}$ & $\mathbf{2 0 0}$ \\
\hline $\mathbf{0}$ & 66 & 66 & 66 & 66 & 66 & 66 \\
\hline $\mathbf{6 0}$ & 64 & 63 & 62 & 59 & 58 & 54 \\
\hline $\mathbf{1 2 0}$ & 57 & 55 & 54 & 54 & 52 & 50 \\
\hline $\mathbf{1 8 0}$ & 55 & 53 & 52 & 51 & 49 & 46 \\
\hline
\end{tabular}

Table (1): Water contact angle of the polystyrene treated with argon and oxygen plasma 


\begin{tabular}{|c|c|c|c|c|c|c|}
\hline \multirow{3}{*}{$\begin{array}{l}\text { Treatment } \\
\text { time } \\
\text { (seconds) }\end{array}$} & \multicolumn{3}{|c|}{$\begin{array}{c}\text { Argon plasma treated } \\
\text { Microwave power (watt) }\end{array}$} & \multicolumn{3}{|c|}{$\begin{array}{l}\text { Oxygen plasma treated } \\
\text { Microwave power (watt) }\end{array}$} \\
\hline & 100 & 150 & 200 & 100 & 150 & 200 \\
\hline & 100 & 150 & 200 & 100 & 150 & 200 \\
\hline $\mathbf{0}$ & 66 & 66 & 66 & 66 & 66 & 66 \\
\hline 60 & 64 & 63 & 57 & 52 & 49 & 46 \\
\hline 120 & 62 & 54 & 51 & 44 & 40 & 36 \\
\hline 180 & 58 & 51 & 47 & 41 & 36 & 35 \\
\hline
\end{tabular}

Table (2): Water contact angle of the polyethylene treated with argon and oxygen plasma 